\title{
DEVELOPMENT OF ATLID RETRIEVAL ALGORITHMS
}

\author{
D.P. Donovan ${ }^{*}$, , G-J van Zadelhoff ${ }^{1}$, J. E. Williams ${ }^{1}$, U. Wandinger ${ }^{2}$, M. Haarig ${ }^{2}$, Z. $\mathbf{Q u}^{3}$ \\ ${ }^{1}$ Royal Netherlands Meteorological Institute (KNMI), Utrechtseweg 297, PO Box 201, \\ 3730 AE de Bilt, the Netherlands \\ ${ }^{2}$ Leibniz Institute for Tropospheric Research. Permoserstraße 15. 04318 Leipzig, Germany. \\ ${ }^{3}$ Environment and Climate Change (MRD), Downsview, Ontario, Canada. \\ *Email:Donovan@knmi.nl
}

\begin{abstract}
ATLID ("ATmospheric LIDar") is the lidar to be flown on the multi-instrument Earth Clouds and Radiation Explorer (EarthCARE or ECARE) joint ESA/JAXA mission now scheduled for launch in 2022. ATID is a 3 channel linearly polarized High-Spectral Resolution (HSRL) system operating at $355 \mathrm{~nm}$. Cloud and aerosol optical properties are key ECARE products. This paper will provide an overview of the ATLID L2a (i.e. single instrument) retrieval algorithms being developed and implemented in order to derive cloud and aerosol optical properties.
\end{abstract}

\section{INTRODUCTION}

EarthCARE[1] is a cloud-aerosol-precipitationradiation process oriented mission. In addition to ATLID, the instrument suite includes, a $94 \mathrm{GHz}$, Doppler Cloud Radar supplied by Japan (CPR), a Multispectral Cloud/Aerosol imager for narrowband TOA radiances (MSI) and a 3-view BroadBand Long- and Short-Wave Radiometer for TOA radiance (BBR) measurements and flux estimation.

ATLID is a linearly polarized three channel lidar operating at $355 \mathrm{~nm}$. The vertical resolution of the return signal is $100 \mathrm{~m}$ throughout most of the atmosphere. The PRF is $51 \mathrm{~Hz}$ and nominally two shots will be averaged on-board giving a horizontal resolution on the order of $275 \mathrm{~m}$. The lidar delivers profiles of the parallel, Mie (or particulate) attenuated backscatter, the parallel Rayleigh (molecular) attenuated backscatter and the corresponding total (Mie+Rayleigh) crosspolarized return. More details can be found in [2].

A comprehensive suite of retrieval algorithms is being developed for EarthCARE As outlined in [1], both (primarily) single-instrument (L2a) and synergistic multi-instrument (L2b) algorithms are being developed. In this paper, the focus is on the L2a ATLID algorithms.

\section{THE L2a ATLID ALGORITHMS}

In this Section, the ECARE L2a lidar algorithms are briefly described in turn.

\subsection{ATLID Featuremask (A-FM).}

A-FM uses a combination of image processing techniques in order to identify regions of clouds/aerosols, surface returns, clear air, or attenuated regions. The detected aerosol/cloud regions are separated into cloud phase and aerosol type later in subsequent processing steps.

\subsection{Aerosol oriented extinction and backscatter retrieval routine (A-AER).}

This procedure uses conventional HRSL retrieval methods for determining extinction and backscatter at the $50 \mathrm{~km}^{+}$horizontal scale (e.g. deriving the extinction based on the log derivative of the Rayleigh signal). In order to do this the lidar signals must be appropriately masked and averaged to achieve a target SNR. The averaging mask originates from the A-FM output which is used to avoid averaging "strong" and "weak" features together.

\subsection{Cloud and aerosol Extinction, Backscatter and Depolarization procedure (A-EBD).}

This routine retrieves the aerosol and cloud extinction and backscatter profiles at the $1-\mathrm{km}$ horizontal scale. At this scale the SNR of the molecular scattering channel return is too low to enable the techniques employed by the A-AER approach. Instead, the method relies on finding the optimal profile of the extinction-to-backscatter ratio $(S)$ that allows one to invert the total lidar signal to produce an extinction profile consistent with the observed Rayleigh channel signal. Multiple-scattering (MS) effects which are important for accurate cloud (and in some cases aerosol) retrievals are included in the procedure which is cast in an optimal-estimation framework.. 
As a-priori information, the $\mathrm{S}$ estimates produced by A-AER are used.

\subsection{Target classification procedure (A-TC).}

A-TC uses extinction, backscatter and depolarization ratio, as well as auxiliary inputs such as ECMWF forecast temperature, in order to classify targets into classes such as water or ice cloud or aerosol type. The aerosol typing scheme is based primarily on using the $\mathrm{S}$ and particle depolarization ratio to assign a class to the aerosol [3]. The cloud phase determination scheme uses backscatter and depolarization in a manner similar to that employed for the CALIOP retrievals.

\subsection{ATLID Cloud Top Height (A-CTH)}

The A-CTH procedure actually detects all recoverable significant boundaries. The cloud top height itself is defined as the upper geometrical boundary of the uppermost cloud layer in the atmosphere. The A-CTH product is derived from the Mie co-polar signal by searching for characteristic signal gradients using a wavelet covariance transform approach [4]. Depending on the cloud optical thickness and the signal-tonoise ratio, the boundaries are detected with a horizontal resolution of 1-km (thick clouds) or $11 \mathrm{~km}$ (thin clouds). The product contains a simplified classification of the uppermost cloud as well as quality indicators in terms of level of confidence of the detection and level of consistency with the A-TC product.

\subsection{ATLID Aerosol Layer Descriptor (A-ALD)}

The A-ALD product contains geometrical and optical information on aerosol layers. The same wavelet transform algorithm as used in the A$\mathrm{CTH}$ retrieval is used to derive aerosol layer boundaries from the Mie co-polar signal, averaged over about $10 \mathrm{~km}$ horizontally. Appropriate threshold settings allow for the discrimination of clouds and aerosol. The A-ALD product provides the upper and lower geometrical boundaries of significant aerosol layers, the optical thickness of each layer (ALOT), the column and the stratospheric AOT at $355 \mathrm{~nm}$. ALOT and AOT are calculated by integrating the extinction profile taken from the A-EBD product. In addition, layermean values of extinction and backscatter coefficient, lidar ratio and particle linear depolarization ratio are calculated from the AEBD product. The A-ALD product is defined for cloud-free conditions only. It contains quality indicators in terms of level of confidence for the aerosol layer detection and level of consistency with the A-TC product.

\section{ALGORITHM PROCESS \\ DEVELOPMENT}

A-Train observations are playing important roles within the ECARE algorithm development process. However, given the wavelength difference and the non-HSRL nature of the instrument, CALIPSO observations are not sufficient for ATLID algorithm development purposes. Accordingly, a number of simulated realistic scenes have been developed and simulated L1 data sets have been created using the multiple-scatter lidar models contained within the EarthCARE simulator framework [5].
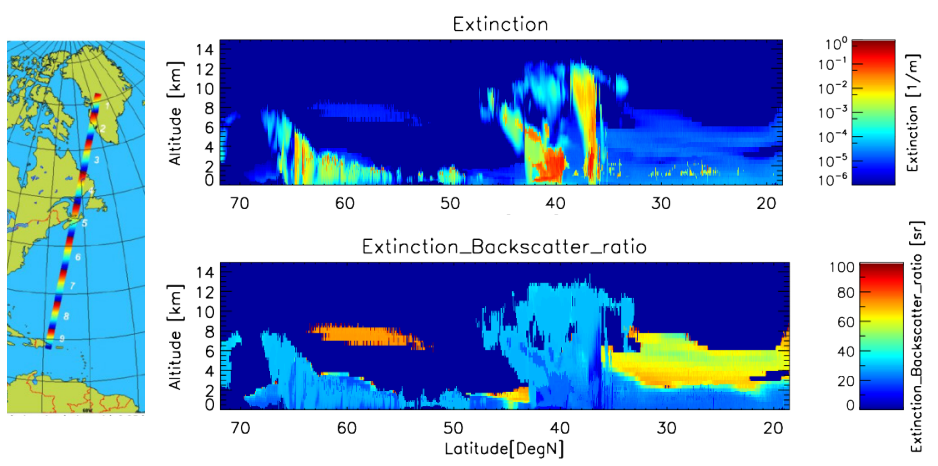

Figure 1: Illustration of the Halifax scene swath and the seven high resolution $(0.25 \mathrm{~km}$ horizontal resolution) sections comprising the scene (Left). Corresponding derived vertical nadir slices of the $355 \mathrm{~nm}$ extinction and backscatter-to-extinction ratio fields (Right).

One such scene (the so-called Halifax scene) is depicted in Figure 1. The scene is built around high-resolution segments of the environment Canada GEM forecast model [6]. The GEM model uses a dual moment micro-physical cloud scheme covering multiple hydrometers (cloud ice, snow, cloud water rain, grauple and hail). Using these fields as a foundation and imposing realistic particle habits and size distributions, the cloud/hydrometer fields necessary for the lidar simulations were created. Aerosol information did not come from the GEM model data. Rather, colocated aerosol CAMS aerosol fields [7] were used to construct the necessary aerosol fields. 
Simulated fields of L1 attenuated backscatter for the three ATLID channels are shown in Figure 2.
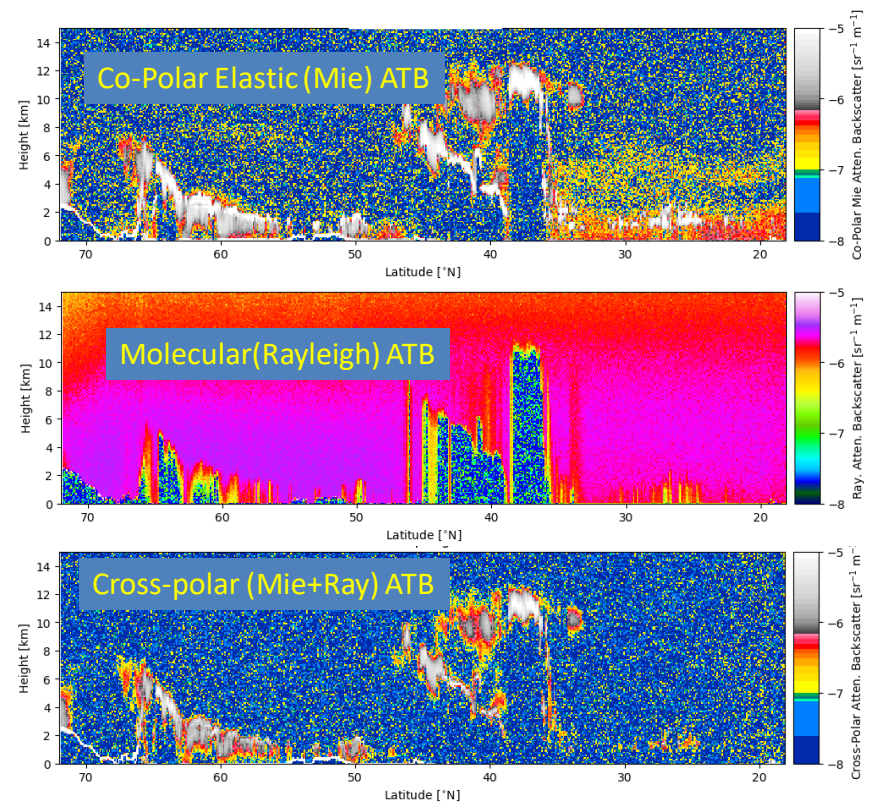

Figure 2: Simulated Attenuated backscatter for the Copolar elastic (Mie), co-polar molecular (Rayleigh) and total cross-polar ATLID channels corresponding to the Halifax scene.

\section{EXAMPLE RESULTS}

In this section various illustrative sample results from the algorithms previously introduced will be presented.

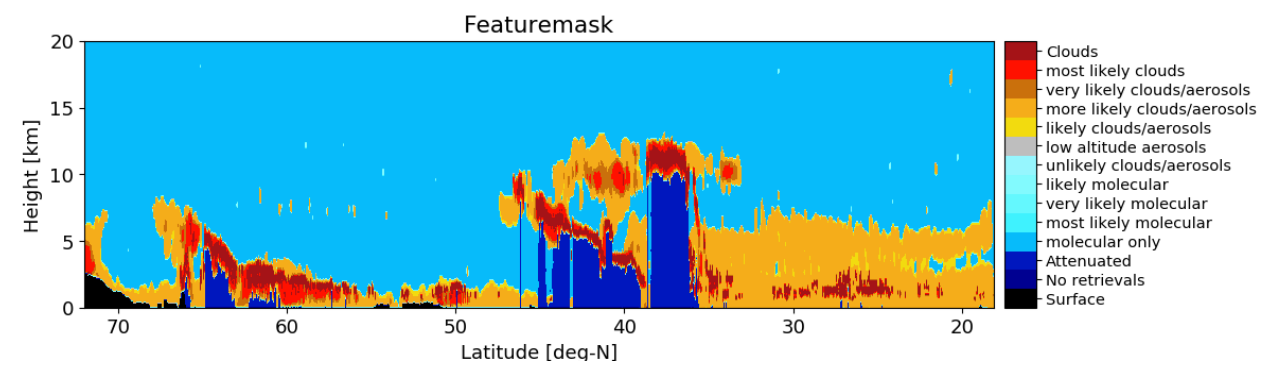

Figure 3: A-FM output for the Halifax Scene.

\subsection{A-FM}

The main output generated by A-FM for the Halifax scene is shown in Figure 3. Here the Red colors correspond to confidently detected strong features while the light-Blue areas correspond to confidently detected clear air regions. At this stage, the feature classes ((likely cloud, aerosol, likely clear etc.) assigned to the level of target detection confidence are preliminary and are intended to be interpreted in a qualitative sense.
A more robust and accurate classification is performed in the following processing steps.
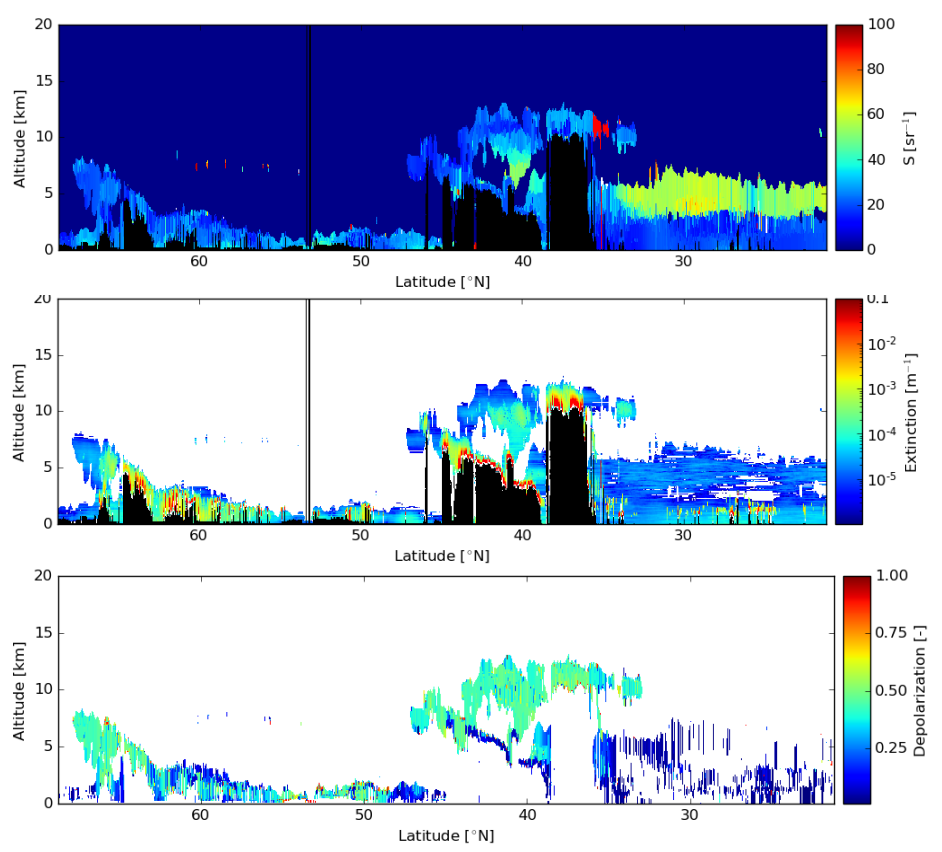

Figure 4: A-EBD results for the Halifax scene. Shown are the retrieved backscatter-to-extinction ratios (Top), the cloud and aerosol extinction fields (Middle) and the cloud/aerosol depolarization ratio (Bottom).

\subsection{A-EBD}

Sample output fields from the A-EBD algorithm are shown below. As discussed earlier the A-EBD

algorithm uses low resolution input aerosol and "thin" ice clouds S fields from the A-AER algorithm (not shown) as well as a-priori values for the cloud $\mathrm{S}$ values in order to produce merged results for both clouds and aerosols. The data shown are actually a composite of two horizontal resolutions. For the "strong" features the horizontal resolution is 1-km while for the "weak" features it is $121 \mathrm{~km}$.

The S and extinction fields are, generally, well retrieved (an exception being the high $\mathrm{S}$ values at around $14 \mathrm{~km}$ at about $35^{\circ} \mathrm{N}$ ). Quantitative statistical evaluations show that e.g. that typical aerosol extinction profiles with a horizontal resolution of $121 \mathrm{~km}$ and a vertical resolution of $0.1 \mathrm{~km}$ are retrieved with an RMS accuracy within about $20 \%$. 


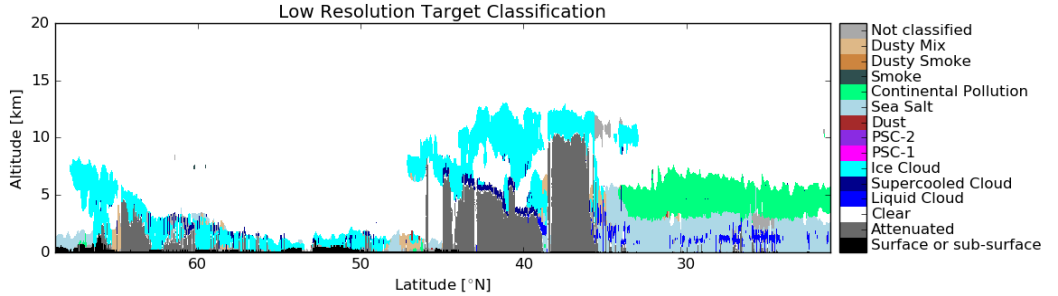

measurements will confront the algorithm development teams with surprises. However, the extensive realistic simulations will have certainly helped ensure that highquality products from EarthCARE will be generated quickly after real

Figure 5: Target classification corresponding to the Halifax scene.

\subsection{A-TC}

Compared to A-FM, A-TC is able to draw on more information (i.e. attenuated backscatter only in the case of A-FM while attenuated backscatter, particle depolarization, particle extinction and extinction-to-backscatter are all used by A-TC) to produce a more accurate and detailed classification of the clouds and aerosols present.

The A-TC output corresponding to the Halifax scene is shown in Figure 5. As was the case for the data shown in Figure 4 the cloud classification has a horizontal resolution of $1 \mathrm{~km}$ while the aerosol classification field corresponds to a horizontal resolution of $120 \mathrm{~km}$. Here it can be seen that the cloud/aerosol discrimination is robust and a good separation between the boundary-layer marine aerosol field and the lofted aerosol layer is achieved.

\subsection{A-CTH}

Sample A-CTH output is shown in Figure 6. Here a "zoomed in" cloud dominated section of the Halifax scene is presented in order to better illustrate the output. A-CTH uses a wavelet technique to detect significant layers and so compliments the A-FM based approach.

One of the main intended uses of A-CTH data is in synergy with the MSI also mounted on EarthCARE. To this end, A-CTH provides not only the detected cloud-top-height but also information on the number of detected significant layers. This is illustrated by the various colored lines present in Figure 6.

\section{SUMMARY}

The launch of EarthCARE at this point remains in the future. However, given the complexity of the processing chain and the novel nature of ATLID data the intervening time is being put to good use. There is no doubt that the real ATLID observations have been acquired.

\section{ACKNOWLEDGEMENTS}

The work presented in this paper is performed under the APRIL project funded by ESA (contract 4000112018/14/NL/CT).

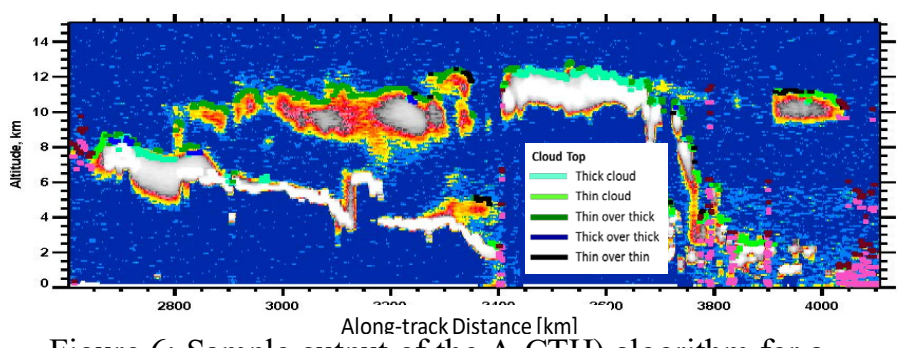

Figure 6: Sample output of the A-CTH) algorithm for a sub-section (approx. 33 to $47^{\circ} \mathrm{N}$ ) of the Halifax scene.

\section{REFERENCES}

[1] Illingworth, A., et al., 2014: THE EARTHCARE SATELLITE: The next step forward in global measurements of clouds, aerosols, precipitation and radiation. Bull. Am. Met. Soc. 96, 1311-1332.

[2] do Carmo, J.P., et al., 2016: ATLID, ESA Atmospheric LIDAR Developement Status, Proc. ILRC27,DOI:https://doi.org/10.1051/epjconf/20161190 4003

[3] Wandinger U., et al., 2016: HETEAC: The Aerosol Classification Model for EarthCARE, Proc. ILRC27, DOI:10.1051/epjconf/201611901004

[4] Brooks, I., 2003: Finding Boundary Layer Top: Application of a wavelet covariance transform to lidar backscatter profiles, J. Atmos. Ocean. Tech. 20, 10921105.

[5] Donovan, D. P., et al., 2015: A depolarisation lidarbased method for the determination of liquid-cloud microphysical properties, Atmos. Meas. Tech., 8, 237266, https://doi.org/10.5194/amt-8-237-2015.

[6] Côté, J., etl al., , 1998: The operational CMC-MRB

Global Environmental Multiscale (GEM) model: Part I

- Design considerations and formulation, Mon.Wea. Rev., 126, 1373-1395.

[7] Inness, A., et, al., 2019: The CAMS reanalysis of atmospheric composition, Atmos. Chem. Phys., 19, 3515-3556, https://doi.org/10.5194/acp-19-3515-2019. 